\title{
Energizing depression research
}

$\mathrm{M}$ akers of antidepressant drugs may have been tempted to become their own best customers recently. Allegations that GlaxoSmithKline, which produces the popular antidepressant Paxil, suppressed negative clinical trial results on antidepressant use in young people has led to legal action and public outcry. No wonder-not only did the results suggest that Paxil was not as effective as the company had advertised, but they also revealed a potentially dangerous drug-associated increase in suicidal behavior. The controversy highlights a general problem in the treatment of depression: current antidepressant drugs are far from perfect.

Part of the problem is that the serendipitous discovery of antidepressant drugs has dictated much of what we know about the underlying neurobiology of depression. Although depression has been recognized since antiquity, it was not until the middle of the last century that drugs that elevate brain norepinephrine and serotonin (also known as monoamines) were found to be effective antidepressants. This realization led to the monoamine hypothesis of depression, which has shaped thinking in the field and drug development ever since. However, evidence in support of the hypothesis is weak at best. Perhaps most troubling is that antidepressant drugs affect monoamine levels within hours, yet take weeks to improve a patient's mood, suggesting that their effect depends critically on some adaptive response of the brain to the drugs. And for a large proportion of patients (as much as $30 \%$ by some estimates), currently available drugs are no help at all.

Today's most promising targets for effective antidepressants are likely to lie downstream of the monoamines, explains Ronald Duman at Yale University. He and others have found that antidepressants increase the production of neurotrophins, and that delivery of these growth factors to the brains of animals can mimic the behavioral effects of antidepressants, suggesting that depression may involve changes in cell survival or plasticity. Added support for the idea comes from work showing that antidepressants increase neurogenesis, which Rene Hen at Columbia University has found is necessary for the behavioral effects of these drugs in rodents. Stress, too, seems linked to depression, given that antidepressants suppress the stress response in both human and animal studies. Pharmaceutical companies are now testing the antidepressant actions of compounds that block corticotropin-releasing hormone signaling, the main trigger for stress responses. Although these new ideas are promising, it is unclear whether they will translate into new clinical treatments. "We're a long way from understanding the relationship between theory and treatment response," notes John March, Chief of Child and Adolescent Psychiatry at Duke University Medical Center.

Another complicating factor is that depression probably arises from many different causes. Thus we need to learn how to recognize which treatment is appropriate for a particular individual. "When you look at 100 depressed patients in a study, there are probably 10 different causes," says Duman, who feels that the answer lies in examining depression in families, where the genetics and other determining factors are somewhat more homogenous. Animal models of depression may help to uncover the underlying causes of the disorder, but they are limited because we can only interpret what animal behavior means for emotionality in humans. In particular, the common animal models used to test antidepressant effectiveness, such as learned helplessness resulting from inescapable stress, have been developed based on behavioral responses to known antidepressants, and may not capture the full range of human depression symptoms. "We haven't gotten close to finding what the actual genes are," admits Duman.

We will also need to learn more about the brain circuits that regulate mood normally in order to fix them when they go awry. "If you don't have the circuits and you don't know what cells are important, it is very hard to make progress," says Steven Hyman at Harvard University, who was director of the US National Institute of Mental Health (NIMH) from 1996-2000. Brain imaging studies may help. For example, work from Helen Mayberg at the University of Toronto has shown that metabolic activity in the rostral anterior cingulate predicts whether a patient will respond to antidepressant treatment. Further work to understand the cortico-limbic areas that show activity changes during depression and after drug treatment should help to focus the field on potentially important brain circuitry.

Finally, developing better biomarkers for diagnosing depression will be important to allow doctors to treat patients more selectively (and effectively) at earlier stages of the illness. Physicians currently start someone on a drug, wait six weeks, and then try another drug if the first choice was ineffective. Positive results can therefore take months, an eternity for someone suffering from depression. "Wouldn't it be great if you could diagnose depression the way you would diagnose heart disease?" says Thomas Insel, current Director of the NIMH. Researchers are also trying to develop drugs that work faster and more effectively than those currently available. Under Insel's direction, the NIMH is now undergoing a major reorganization to focus on translational research. "We have to continue to build a basic science commitment, but build it with an eye to what is really important," says Insel.

The World Health Organization estimates that 121 million people worldwide suffer from depression, making it one of the leading causes of disability. Although current drug treatments are effective for many patients, there remains substantial room for improvement. Achieving this goal will require a concerted effort on the part of researchers to tackle the hard questions regarding the neurobiological basis of depression and then to move these theories into the clinic. In doing so, we hope that the field will not lose sight of the importance of basic research to understand the disorder, which will be crucial to the intelligent development of effective treatments. 